\title{
Posterior Malleolar Stabilization of Syndesmotic Injuries is Equivalent to Screw Fixation
}

\author{
Anna N. Miller MD, Eben A. Carroll MD, \\ Robert J. Parker BS, David L. Helfet MD, \\ Dean G. Lorich MD
}

Received: 10 February 2009/Accepted: 15 September 2009/Published online: 2 October 2009

(C) The Association of Bone and Joint Surgeons (B) 2009

\begin{abstract}
Background Fixation of unstable ankle fractures, including fixation of posterior malleolus fracture fragments with the attached, intact posteroinferior tibiofibular ligament (PITFL), reportedly provides more stable fixation than transsyndesmotic screws.

Questions/Purposes To confirm this observation we compared the Foot and Ankle Outcome Score (FAOS) and radiographic maintenance of fixation for fractures treated through direct posterior malleolar fixation versus syndesmotic screw fixation.

Methods We prospectively followed 31 one patients with unstable ankle fractures treated with (1) open posterior malleolus fixation whenever the posterior malleolus was fractured, regardless of fragment size (PM group; $n=9$ ); (2) locked syndesmotic screws in the absence of a posterior malleolar fracture ( $\mathrm{S}$ group; $\mathrm{n}=14$ ); or (3) combined
\end{abstract}

Each author certifies that he or she has no commercial associations (eg, consultancies, stock ownership, equity interest, patent/licensing arrangements, etc) that might pose a conflict of interest in connection with the submitted article.

Each author certifies that his or her institution approved the human protocol for this investigation, that all investigations were conducted in conformity with ethical principles of research, and that informed consent for participation in the study was obtained.

This work was performed at the Hospital for Special Surgery, New York, NY, USA.

A. N. Miller ( $₫)$, R. J. Parker, D. L. Helfet, D. G. Lorich Hospital for Special Surgery, 535 East 70th Street, New York, NY, USA

e-mail: millera@hss.edu

E. A. Carroll

Wake Forest University School of Medicine, Winston-Salem, NC, USA fixation in fracture-dislocations and more severe soft tissue injury (C group; $n=8$ ). All patients had preoperative MRI confirming syndesmotic injury and an intact PITFL; postoperative and followup radiographs were evaluated for syndesmotic congruence. The minimum followup was 12 months (mean, 15 months; range, 12-31 months).

Results Postoperative and followup FAOS scores were similar in the three groups. The tibiofibular clear space was greater in the S versus the PM group, but we found no other differences in the postoperative versus followup measurements between the PM, S, and C groups.

Conclusions Syndesmotic fixation through the posterior malleolus and PITFL is maintained at followup, and these patients have functional outcomes at least equivalent to outcomes for patients having syndesmotic screw fixation. Level of Evidence Level II, therapeutic study. See Guidelines for Authors for a complete description of levels of evidence.

\section{Introduction}

Ankle fractures are among the most common lower extremity injuries. Displaced fractures typically are treated operatively to restore anatomic alignment of joint surfaces, reduce tibiotalar contact stresses, and minimize posttraumatic arthritis $[1,2,14,24,25,28,29,32]$. Treatment of these fractures must include intraoperative examination of the syndesmosis to ensure its integrity. Injury to the syndesmotic ligament complex can lead to disruption of the ankle mortise. Furthermore, injury to the syndesmosis may occur with many types of ankle fractures, not just AO Weber Type $\mathrm{C}$ with which it traditionally has been associated $[12,17,21,23,27,31]$. 
In a previous study, syndesmotic instability treated with traditional transsyndesmotic fixation methods was found to have a $52 \%$ rate of syndesmotic malreduction as evaluated by CT [11]. In that study, ankle fractures with syndesmotic injuries were treated in a typical fashion, first addressing medial and lateral malleolar fractures with open reduction and internal fixation and then addressing the syndesmosis reduction and stabilization with fluoroscopic reduction and observation of transsyndesmotic screw placement. We routinely removed syndesmosis screws, requiring another surgical procedure [6].

Our biomechanical study suggested fixation of the posterior malleolus fracture restores more stability to the syndesmosis than transsyndesmotic fixation alone [10]. Studies using preoperative MRI to evaluate ankle fractures suggest fracture patterns that include an associated posterior malleolar fracture reliably have an intact posteroinferior tibiofibular ligament (PITFL) [10, 11]. Ogilvie-Harris et al. showed the PITFL alone makes up $42 \%$ of the strength of the syndesmosis [22]. Based on this information, cadaveric models have been used to evaluate stabilization of the syndesmosis through the PITFL. After the PITFL was stabilized by direct open reduction and internal fixation of its attached posterior malleolar fracture fragment, stability was greater than with syndesmotic screws alone [10].

Therefore, we subsequently established a new protocol to stabilize the ankle syndesmosis with a more anatomic reconstruction of the tibial incisura. With this reconstruction we used open reduction and internal fixation of all posterior malleolar fractures and used syndesmotic screw fixation only when a posterior malleolus fracture was not present or with major soft tissue trauma such as a fracturedislocation of the ankle (see Materials and Methods). The new protocol involved direct observation of syndesmosis reduction in all cases with fixation concentrated on centering the fibula in the tibial incisura in the sagittal plane if screws were necessary.

To evaluate the new protocol, we: (1) determined differences in FAOS in patients with open posterior malleolus fixation alone, locked syndesmotic screws in the absence of a posterior malleolar fracture, and combined fixation; (2) evaluated syndesmosis reduction and confirmed maintenance of fixation radiographically; and (3) determined whether this more direct means of syndesmotic reconstruction would maintain radiographic measurements and attain equivalent functional outcomes as patients treated with syndesmotic screw fixation.

\section{Materials and Methods}

We retrospectively reviewed the charts and radiographs of 38 prospectively followed patients who had open reduction and internal fixation of an ankle fracture and syndesmotic injury during a 3-year period (2006-2008). Seven of the 38 patients $(18 \%)$ had not returned for at least 1 year of followup, and were excluded from our study. Thirty-one of the 38 patients $(82 \%)$ had functional surveys and the full radiographic complement of studies. The average age of the patients was 48 years (range, $21-83$ years); there were 20 female and 11 male patients. All patients with ankle fractures had a preoperative MRI indicated and paid for through our institutional research protocol. These preoperative MR images are used to confirm bony and soft tissue injuries consistent with syndesmotic disruption, including interosseous membrane tears greater than $4 \mathrm{~cm}$ proximal to the ankle. MRI also confirmed posterior malleolar fracture with an intact posteroinferior tibiofibular ligament. All patients were admitted through the emergency department after examination and stress radiography as indicated by an orthopaedic resident before any additional studies were ordered. Minimum followup was 12 months (mean, 15 months; range, 12-31 months). This study had prior approval of our Institutional Review Board.

All 31 fractures were classified according to the LaugeHansen system [17]; this classification system refers to a cadaver study in which Lauge-Hansen first placed the foot in position (the first term in the classification name) and then applied external force (the second term in the classification name) to cause certain fracture patterns. In our study, there were 25 supination-external rotation Type IV (80\%) and three pronation-external rotation Type IV (10\%) injuries; three (10\%) were unclassifiable by the LaugeHansen classification as a result of unusual fracture patterns or comminution, mostly in geriatric patients. Eighteen of the 31 patients $(58 \%)$ sustained a posterior malleolus fracture, and eight of these (26\% of total) were fracturedislocations with extensive soft tissue injury. For purposes of selecting treatment, all patients were placed in one of three treatment groups based solely on their fracture pattern. Nine patients with a posterior malleolus fracture were placed in the posterior malleolus group (PM), 14 patients with a syndesmotic injury and no posterior malleolus fracture were placed in the syndesmosis group (S), and eight patients with a known ankle dislocation and posterior malleolus fracture were placed in the combined group (C).

All surgery was performed by one surgeon (DGL). In patients with fracture patterns involving the posterior malleolus (PM and C groups), we restored the ankle syndesmosis by performing open reduction and internal fixation of the posterior malleolar fragment to recreate the posterior tibial incisura. We fixed the posterior malleolus independent of its articular size or percentage of plafond involvement (even when the fragment was too small to convey instability) as long as the intact PITFL remained attached to the posterior malleolar fragment (as confirmed 
with MRI). We used a posterolateral approach to the ankle to treat the posterior malleolar fracture and associated fibular fractures. This approach involves dissection in the intermuscular plane between the peroneus brevis and flexor hallucis longus muscles down to the tibia and fibula. The syndesmosis and posterior malleolus then are exposed by elevating the flexor hallucis longus muscle belly from the posterior aspect of the distal tibia. This approach was chosen for the large muscular flap it leaves over the reconstructed fracture fragments and instrumentation; we believe this is a more physiologic dissection for wound healing, because the incision is closed over muscle instead of bone or hardware.

The posterior malleolar fragment then was reduced anatomically back to the tibia. The posterior malleolar fragment typically was stabilized with a six-hole 2.0 or 2.4 reconstruction, LCP, or T-plate; this served as a buttress or antiglide plate with at least one interfragmentary screw placed across the posterior malleolar fracture plane (Fig. 1).

In patients with ligamentous syndesmotic injury and no posterior malleolar injury, ie, with the PITFL torn and no longer attached to the posterior malleolus (S group), we directly exposed the distal tibiofibular articulation anteriorly using the same posterolateral approach that was used for open reduction and internal fixation of the fibula. The opened articulation then was débrided and cleared of any torn or interposed tissues. We reduced the syndesmosis using a pelvic clamp with the foot in $10^{\circ}$ dorsiflexion. This reduction was performed under direct observation of the distal tibiofibula articulation just proximal to the ankle, attempting to center the fibula in the tibial incisura and confirming fluoroscopic reduction of the syndesmosis using standard radiographic parameters (see subsequently). In addition to the plate fixation used to treat associated fibula fractures, we placed two locked syndesmotic screws through a separate three-hole, locking, one-third tubular plate. The locked plate and screw construct was used to avoid a malreduction force vector that may result from overtightening more traditional cortical screws. The screws were placed through all four cortices (Fig. 2).

Patients with a higher-energy injury involving more soft tissue disruption (ie, fracture-dislocation; C group) had posterior malleolar and syndesmotic fixation (Fig. 3). Patients with fracture-dislocations were defined as those who came in with dislocated tibiotalar joints along with tibial or fibular fractures at presentation to the emergency department or who were grossly unstable at presentation and could not be maintained with the joint reduced.

Before closure, the surgeon assessed all syndesmoses by placing a narrow Hohmann retractor in the interosseous space to apply an external rotation stressor. The distal tibiofibular articulation stability was assessed by direct
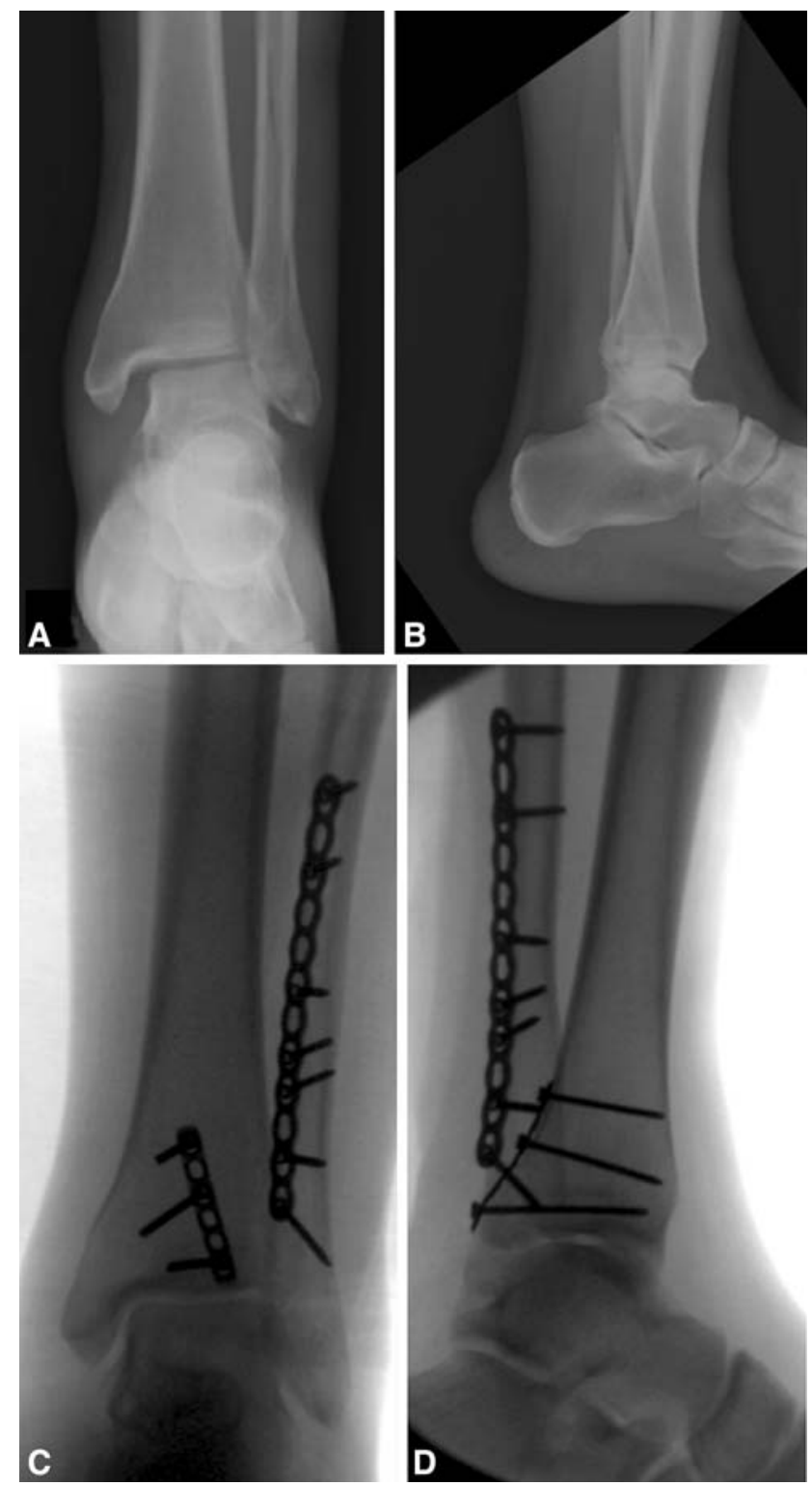

Fig. 1A-D (A) Anteroposterior and (B) lateral radiographs are shown of an injury treated with posterior malleolar fixation. Medial clear space widening and a small posterior malleolus fracture can be seen. (C) Anteroposterior and (D) lateral radiographs obtained after surgery show posterior malleolus stabilization to recreate the tibial incisura. The posterior malleolus and fibula are restored anatomically with plate and screw constructs, and no syndesmotic screws are used.

observation and fluoroscopically. The tibiofibular articulation had less than $1 \mathrm{~mm}$ of diastasis and the superior clear space was not more than $1 \mathrm{~mm}$ different than the medial clear space under stress in all cases; this was our determinant of adequate stabilization [3, 28].

Postoperatively, patients wore a short-leg splint and were instructed not to bear weight for 6 weeks. Subtalar and ankle ROM were instituted in the early postoperative period. Every patient had postoperative bilateral CT scans of the ankles, usually within 48 hours of surgery, to assess 

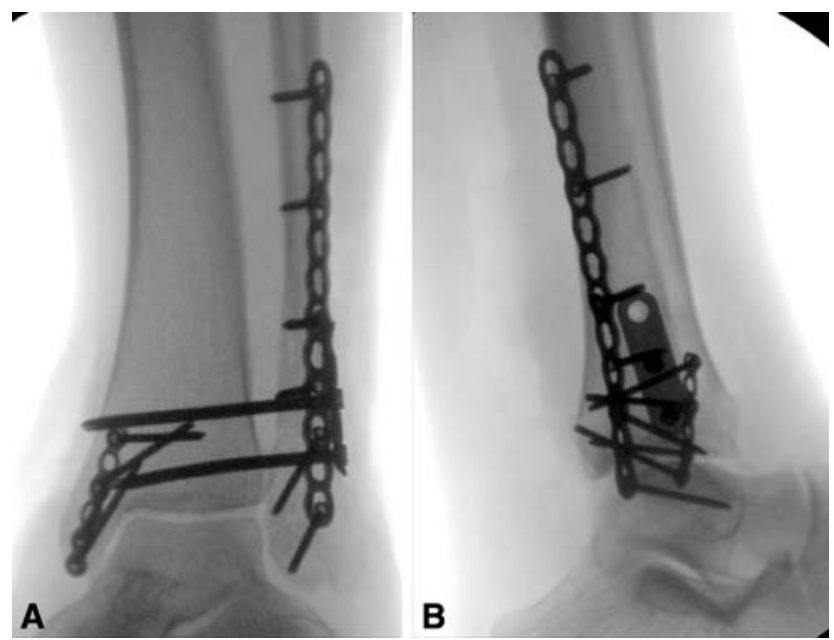

Fig. 2A-B (A) Anteroposterior and (B) lateral radiographs show syndesmotic screw stabilization through a locking one-third tubular plate. In these cases, the medial and lateral malleoli are fixed, and the syndesmosis then is tested. If it still widens under stress, the syndesmotic screw construct is placed.

reduction of the fibula in the incisura as an indicator of tibiofibular joint congruence; this CT also was used to compare the injured ankle with the patient's uninjured, contralateral ankle to determine the quality of syndesmotic reduction. All patients with syndesmotic screws had them removed per our protocol at an average of 4 months after the index surgery.

Patients were followed routinely at 2, 6, 12, and 24 weeks, and 1 year postoperatively. Each patient was given the FAOS (http://www.koos.nu/) [26] version LK1.0 at each followup. The FAOS is a self-administered survey that includes five subscales: pain, symptoms, function in activities of daily living, function in sports and recreation, and overall foot-and-ankle-related quality of life. The survey is designed to include the patient's subjective assessment of his or her function during the week leading up to taking the survey. A normalized score then is calculated for each subscale, ranging from 0 (extreme symptoms) to 100 (no symptoms). The five subsets of the FAOS were evaluated on average and compared among the three fixation groups (S, PM, and C).

At each followup, patients were assessed with AP, lateral, and mortise radiographs to evaluate for syndesmotic reduction, loss of fixation, and hardware failure. Per the methods of Pettrone et al. [23] and Brage et al. [4], three of us (ANM, EAC, RJP) independently made four radiographic measurements on each set of films. The tibiofibular clear space was the horizontal distance from the lateral border of the posterior tibial malleolus to the medial border of the fibula at the point where the posterior malleolus is the widest on the AP view [Fig. 4] [23]. The tibiofibular overlap also was measured on the AP radiograph and is the
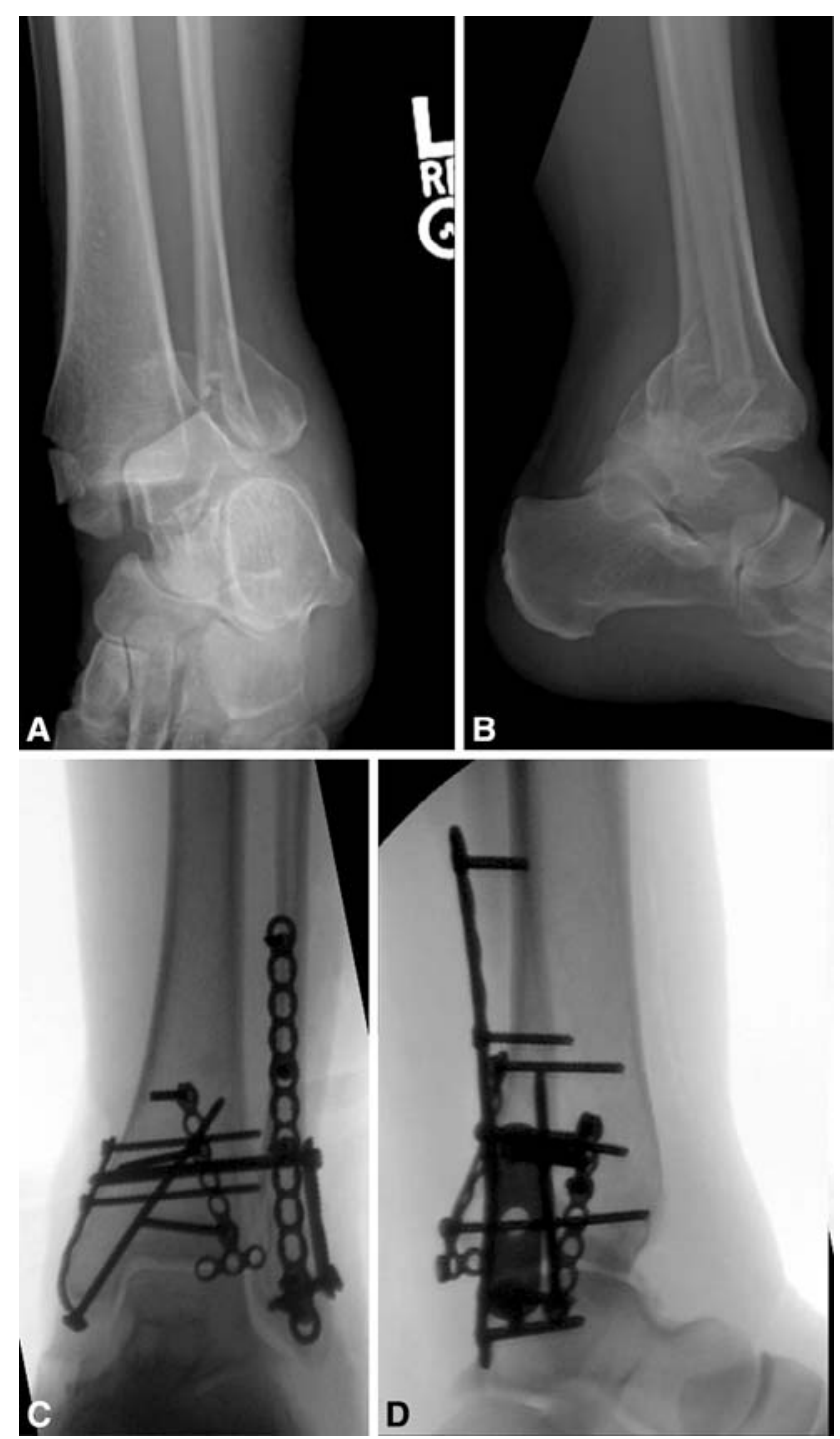

Fig. 3A-D (A) Anteroposterior and (B) lateral radiographs show a fracture-dislocation with complete dislocation and trimalleolar fractures. This is a typical example of a patient treated with combined fixation. (C) Anteroposterior and (D) lateral radiographs show combined posterior malleolar and syndesmotic screw fixation.

horizontal distance between the medial border of the fibula and the lateral border of the anterior tibial prominence (Fig. 4). The third measurement was the tibiofibular overlap as measured on the mortise view. The medial clear space also was measured on the mortise view and was the distance between the lateral aspect of the medial malleolus and the medial border of the talus at the level of the talar dome (Fig. 4). As measurements of syndesmotic congruence, we used the following parameters: tibiofibular clear space less than $6 \mathrm{~mm}$, tibiofibular overlap greater than $6 \mathrm{~mm}$, tibiofibular overlap greater than $1 \mathrm{~mm}$, and medial clear space less than $5 \mathrm{~mm}[5,8,13,16,20,23]$. The mean and standard deviations of the three observers for the radiographic measurements was $0.2 \pm 0.1 \mathrm{~mm}$. The three 


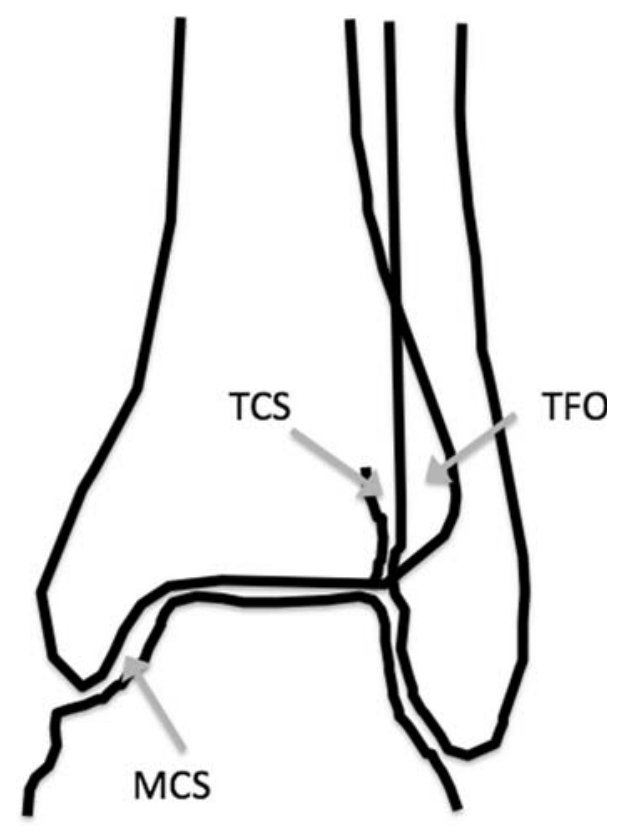

Fig. 4 The drawing shows the measurements as described in the text. TCS $=$ tibiofibular clear space; TFO $=$ tibiofibular overlap; MCS $=$ medial clear space.

observers' numeric data did not differ (ANM versus RJP, $p=0.86 ;$ RJP versus $E A C, p=0.19$; ANM versus $E A C$, $\mathrm{p}=0.44)$.

All analyses compared differences between the three treatment groups (PM, S, and C) using a one-factor ANOVA with Bonferroni post hoc tests. This was used for all five subsets of the FAOS and all four radiographic measurements between preoperative and postsurgical evaluation. All analyses were conducted using SPSS 17.0 for Windows (Chicago, IL).

\section{Results}

The five subsets of the FAOS (pain, symptom, activities of daily living, sports, and foot-and-ankle-related quality of life) were similar among the three groups (Table 1).
Table 1. Foot and Ankle Outcome Score (FAOS) breakdown

\begin{tabular}{lllll}
\hline FAOS subcategory & Average & PM & S & C \\
\hline Pain & 76 & 76 & 78 & 72 \\
Symptom & 69 & 73 & 72 & 58 \\
ADL & 82 & 84 & 79 & 87 \\
Sports & 69 & 65 & 76 & 59 \\
QoL & 55 & 56 & 58 & 49
\end{tabular}

$\mathrm{PM}=$ posterior malleolus syndesmosis reconstruction; $\mathrm{S}=$ syndesmotic screw fixation; $\mathrm{C}=$ combined syndesmotic screw and posterior malleolus fixation; $\mathrm{ADL}=$ activities of daily living; $\mathrm{QoL}=$ footand-ankle-related quality of life.

The mean tibiofibular clear space for the entire cohort of 31 patients was $3.4 \mathrm{~mm}$ postoperatively and $4.1 \mathrm{~mm}$ at latest followup with a net widening of $0.7 \mathrm{~mm}$. The difference in tibiofibular clear space from postoperative to last followup was greater in the S group compared with PM, but this was not statistically significant. The mean tibiofibular overlap, mortise tibiofibular overlap, and medial clear space were similar for the three groups (Table 2).

We observed no radiographic loss of reduction in any of the three groups. The mean net change for the tibiofibular clear space, tibiofibular overlap, mortise tibiofibular overlap, and medial clear space for all of the fixation types was $0 \mathrm{~mm}$ (Table 3). The $\mathrm{S}$ group increased approximately $0.1 \mathrm{~mm}$ for all parameters on average, the PM group decreased $0.3 \mathrm{~mm}$, and the $\mathrm{C}$ group changed $0 \mathrm{~mm}$.

There were no postoperative complications after index surgery or screw removal surgery in any patient; no patients had wound breakdown or infections.

\section{Discussion}

In a previous study, a large percentage of ankle fractures were observed to be malreduced by CT parameters [11]. Our rationale with the current study was to decrease these malreductions by performing fixation based on anatomic posterior malleolar fracture fixation as opposed to

Table 2. Measurements of postoperative and final followup radiographs for each of four radiographic parameters

\begin{tabular}{|c|c|c|c|c|c|c|c|c|}
\hline \multirow[t]{2}{*}{ Radiographic measurement } & \multicolumn{2}{|l|}{ Average } & \multicolumn{2}{|l|}{ PM } & \multicolumn{2}{|l|}{$\mathrm{S}$} & \multicolumn{2}{|l|}{ C } \\
\hline & $\mathrm{P} / \mathrm{O}$ & $\mathrm{F} / \mathrm{U}$ & $\mathrm{P} / \mathrm{O}$ & $\mathrm{F} / \mathrm{U}$ & $\mathrm{P} / \mathrm{O}$ & $\mathrm{F} / \mathrm{U}$ & $\mathrm{P} / \mathrm{O}$ & $\mathrm{F} / \mathrm{U}$ \\
\hline TCS & $3.4 \mathrm{~mm}$ & $4.1 \mathrm{~mm}$ & $3.6 \mathrm{~mm}$ & $4.0 \mathrm{~mm}$ & $3.4 \mathrm{~mm}$ & $4.3 \mathrm{~mm}$ & $3.4 \mathrm{~mm}$ & $4.0 \mathrm{~mm}$ \\
\hline TFO & $7.4 \mathrm{~mm}$ & $6.9 \mathrm{~mm}$ & $6.8 \mathrm{~mm}$ & $6.2 \mathrm{~mm}$ & $7.6 \mathrm{~mm}$ & $7.2 \mathrm{~mm}$ & $7.6 \mathrm{~mm}$ & $7.4 \mathrm{~mm}$ \\
\hline mTFO & $3.9 \mathrm{~mm}$ & $3.3 \mathrm{~mm}$ & $3.1 \mathrm{~mm}$ & $2.1 \mathrm{~mm}$ & $4.0 \mathrm{~mm}$ & $3.9 \mathrm{~mm}$ & $4.4 \mathrm{~mm}$ & $3.7 \mathrm{~mm}$ \\
\hline MCS & $2.8 \mathrm{~mm}$ & $3.1 \mathrm{~mm}$ & $2.6 \mathrm{~mm}$ & $2.9 \mathrm{~mm}$ & $3.0 \mathrm{~mm}$ & $3.2 \mathrm{~mm}$ & $2.7 \mathrm{~mm}$ & $3.1 \mathrm{~mm}$ \\
\hline
\end{tabular}

$\mathrm{PM}=$ posterior malleolus syndesmosis reconstruction; $\mathrm{S}=$ syndesmotic screw fixation; $\mathrm{C}=$ combined syndesmotic screw and posterior malleolus fixation; TCS $=$ tibiofibular clear space; TFO = tibiofibular overlap; $\mathrm{mTFO}=$ mortise tibiofibular overlap; $\mathrm{MCS}=$ medial clear space; $\mathrm{P} / \mathrm{O}=$ postoperative; $\mathrm{F} / \mathrm{U}=$ followup. 
Table 3. Measurement differences between postoperative and final radiographs

\begin{tabular}{lllll}
\hline $\begin{array}{l}\text { Radiographic } \\
\text { measurement }\end{array}$ & Total & PM & S & C \\
\hline TCS & $+0.7 \mathrm{~mm}$ & $+0.4 \mathrm{~mm}$ & $+0.9 \mathrm{~mm}$ & $+0.7 \mathrm{~mm}$ \\
TFO & $-0.5 \mathrm{~mm}$ & $-0.7 \mathrm{~mm}$ & $-0.5 \mathrm{~mm}$ & $-0.2 \mathrm{~mm}$ \\
mTFO & $-0.6 \mathrm{~mm}$ & $-1.1 \mathrm{~mm}$ & $-0.1 \mathrm{~mm}$ & $-0.7 \mathrm{~mm}$ \\
MCS & $+0.3 \mathrm{~mm}$ & $-0.2 \mathrm{~mm}$ & $+0.2 \mathrm{~mm}$ & $+0.4 \mathrm{~mm}$ \\
Average & $0.0 \mathrm{~mm}$ & $-0.3 \mathrm{~mm}$ & $+0.1 \mathrm{~mm}$ & $+0.1 \mathrm{~mm}$
\end{tabular}

$\mathrm{PM}=$ posterior malleolus syndesmosis reconstruction; $\mathrm{S}=$ syndesmotic screw fixation; $\mathrm{C}=$ combined syndesmotic screw and posterior malleolus fixation; TCS $=$ tibiofibular clear space; TFO $=$ tibiofibular overlap; $\mathrm{mTFO}=$ mortise tibiofibular overlap; $\mathrm{MCS}=$ medial clear space.

fluoroscopically reduced syndesmotic screw fixation. We believe direct reduction of posterior malleolar fractures, independent of fragment size, stabilizes the syndesmosis through the intact PITFL, resulting in more anatomic reduction of the distal tibiofibular articulation. This theory was first put forth by Mast et al., in 1980, but previously has not been proven in a methodical fashion [18]. In addition, in a recent meta-analysis, no consensus was found in the literature regarding which fragment sizes of posterior malleolar fractures should be fixed [30]. Our purposes for this study were (1) to compare FAOS scores among three surgical fixation groups; (2) compare radiographic measurements (tibiofibular clear space, tibiofibular overlap, mortise tibiofibular overlap, and medial clear space) to evaluate maintenance of fixation for the three groups; and (3) compare these results between the gold standard, syndesmotic screw fixation, and our anatomic posterior malleolar fixation.

Our study has several limitations. First, we evaluated the syndesmosis reduction only with radiographs. Several studies suggest radiographic evaluations are insensitive when evaluating the syndesmosis [3, 7, 27]. Gardner et al. reported a 52\% malreduction seen on CT as compared with apparently well-reduced fractures on plain radiographs [11]. We also observed that patients who had syndesmotic screws (S group) had a small mean increase in their tibiofibular clear space with time, whereas other radiographic parameters stayed the same. This increase is less than $1 \mathrm{~mm}$; because most surgeons accept $2 \mathrm{~mm}$ as a cutoff for joint incongruency, it is unclear whether this is clinically relevant. In our opinion, this points more to the difficulties of measuring dynamic articulations with static radiographs than a sign of instability. Second, we had a small patient cohort. These small numbers could have led to bias resulting from sample size, especially if there were any major outliers. Third, the followup times were relatively short and a longer followup is needed to confirm that our functional and radiographic results continue to be maintained at longer times. Finally, we acknowledge there is an inherent difficulty in comparing groups of patients with different injury patterns. In the current cohort, we believe the $\mathrm{PM}$ and $\mathrm{S}$ groups were closely comparable because the same mechanism of injury can cause either fracture pattern. However, the $\mathrm{C}$ group, which in most cases the fracture was caused by a higher-energy injury, may not be as comparable. In these cases, because the FAOS scores and radiographic parameters were equivalent to those of the other two groups, we do not believe this caused a change in how our data should be viewed, because these patients most likely would have worse FAOS scores and radiographic parameters as a result of the nature of their injuries.

We found similar FAOS scores among our three groups, whether posterior malleolar, syndesmotic screw, or both types of fixation were used. There are no other published studies examining posterior malleolar fixation of this type; however, one study does show patients with a posterior malleolar fracture of any size (even those small enough to be treated nonoperatively such as those in our current study) tend to give decreased functional outcomes scores [15]. A recent study did show patients have few local wound complications with the posterolateral approach as we used in our study [9].

Several studies have suggested syndesmotic reduction is the most important factor contributing to functional outcome $[16,20,31]$. An anatomic reconstruction of the posterior malleolus and PITFL complex for syndesmosis injury is reportedly more accurate than syndesmotic screw fixation and indirect reduction [19]. Without recreation of the posterior incisura as a posterior buttress, the fibula may rotate out the back of the articulation, which can be difficult to appreciate on plain radiographs. Our data suggest reduction through the posterior malleolus and PITFL complex is as stable radiographically at followup as syndesmotic screw stabilization.

In comparing our cohort of anatomic posterior malleolar incisura recreation with a group treated through the gold standard, syndesmotic screw fixation, we have seen that the patients are not considerably different functionally or radiographically. In addition to the lack of true anatomic reduction when using syndesmotic screws, there are many problems associated with the screws, including the fact that they sometimes are removed at a later date. This entails a second procedure for the patient, which has its own inherent risk factors. Leaving screws in place can lead to many other problems, including loosening, screw fatigue fracture, syndesmosis widening, tibiofibular synostosis, and osteolysis [6]. Our data suggest patients have equivalent outcomes using posterior malleolar fixation instead of syndesmotic screw stabilization, bypassing this controversy entirely. 
Anatomic fixation of the posterior malleolus, stabilizing the syndesmosis through the PITFL, was biomechanically superior to syndesmotic screw fixation in a cadaver study [10]. Our data suggest patients treated in this manner retain fixation and alignment at followup. Meticulous reduction of the posterior malleolus and PITFL complex to recreate the tibial incisura led to short-term results equivalent to syndesmotic screws radiographically and functionally. Also because, in some institutions, syndesmotic screws are removed routinely through a separate operative procedure, posterior malleolar fixation saves patients the morbidity of another trip to the operating room. When a posterior malleolar fracture is present, we recommend anatomic reconstruction, regardless of the size of the fracture fragment, to recreate the incisura; this obviates the need for syndesmotic screws.

Acknowledgments We thank Dr. Stephen Lyman for work on the statistical portion of our study and Omesh Paul for assistance with data collection.

\section{References}

1. Ali MS, McLaren CA, Rouholamin E, O'Connor BT. Ankle fractures in the elderly: nonoperative or operative treatment. J Orthop Trauma. 1987;1:275-280.

2. Bauer M, Jonsson K, Nilsson B. Thirty-year follow-up of ankle fractures. Acta Orthop Scand. 1985;56:103-106.

3. Beumer A, van Hemert WL, Niesing R, Entius CA, Ginai AZ, Mulder PG, Swierstra BA. Radiographic measurement of the distal tibiofibular syndesmosis has limited use. Clin Orthop Relat Res. 2004;423:227-234.

4. Brage ME, Bennett CR, Whitehurst JB, Getty PJ, Toledano A. Observer reliability in ankle radiographic measurements. Foot Ankle Int. 1997;18:324-329.

5. Burns WC 2nd, Prakash K, Adelaar R, Beaudoin A, Krause W. Tibiotalar joint dynamics: indications for the syndesmotic screw-a cadaver study. Foot Ankle. 1993;14:153-158.

6. Dattani R, Patnaik S, Kantak A, Srikanth B, Selvan TP. Injuries to the tibiofibular syndesmosis. $J$ Bone Joint Surg Br. 2008;90:405-410.

7. Ebraheim NA, Lu J, Yang H, Mekhail AO, Yeasting RA. Radiographic and CT evaluation of tibiofibular syndesmotic diastasis: a cadaver study. Foot Ankle Int. 1997;18:693-698.

8. Ebraheim NA, Mekhail AO, Gargasz SS. Ankle fractures involving the fibula proximal to the distal tibiofibular syndesmosis. Foot Ankle Int. 1997;18:513-521.

9. Forberger J, Sabandal PV, Dietrich M, Gralla J, Lattmann T, Platz A. Posterolateral approach to the displaced posterior malleolus: functional outcome and local morbidity. Foot Ankle Int. 2009;30:309-314.

10. Gardner MJ, Brodsky A, Briggs SM, Nielson JH, Lorich DG. Fixation of posterior malleolar fractures provides greater syndesmotic stability. Clin Orthop Relat Res. 2006;447:165-171.

11. Gardner MJ, Demetrakopoulos D, Briggs SM, Helfet DL, Lorich DG. Malreduction of the tibiofibular syndesmosis in ankle fractures. Foot Ankle Int. 2006;27:788-792.

12. Gardner MJ, Demetrakopoulos D, Briggs SM, Helfet DL, Lorich DG. The ability of the Lauge-Hansen classification to predict ligament injury and mechanism in ankle fractures: an MRI study. J Orthop Trauma. 2006;20:267-272.

13. Harper MC. An anatomic and radiographic investigation of the tibiofibular clear space. Foot Ankle. 1993;14:455-458.

14. Hughes JL, Weber H, Willenegger H, Kuner EH. Evaluation of ankle fractures: non-operative and operative treatment. Clin Orthop Relat Res. 1979;138:111-119.

15. Jaskulka RA, Ittner G, Schedl R. Fractures of the posterior tibial margin: their role in the prognosis of malleolar fractures. J Trauma. 1989;29:1565-1570.

16. Kennedy JG, Soffe KE, Dalla Vedova P, Stephens MM, O’Brien T, Walsh MG, McManus F. Evaluation of the syndesmotic screw in low Weber C ankle fractures. J Orthop Trauma. 2000;14:359366.

17. Lauge-Hansen N. Fractures of the ankle. II. Combined experimental-surgical and experimental-roentgenologic investigations. Arch Surg. 1950;60:957-985.

18. Mast JW, Teipner WA. A reproducible approach to the internal fixation of adult ankle fractures: rationale, technique, and early results. Orthop Clin North Am. 1980;11:661-679.

19. Miller AN, Carroll EA, Parker RJ, Boraiah S, Helfet DL, Lorich DG. Direct visualization for syndesmotic stabilization of ankle fractures. Foot Ankle Int. 2009;30:419-426.

20. Mont MA, Sedlin ED, Weiner LS, Miller AR. Postoperative radiographs as predictors of clinical outcome in unstable ankle fractures. J Orthop Trauma. 1992;6:352-357.

21. Nielson JH, Gardner MJ, Peterson MG, Sallis JG, Potter HG, Helfet DL, Lorich DG. Radiographic measurements do not predict syndesmotic injury in ankle fractures: an MRI study. Clin Orthop Relat Res. 2005;436:216-221.

22. Ogilvie-Harris DJ, Reed SC, Hedman TP. Disruption of the ankle syndesmosis: biomechanical study of the ligamentous restraints. Arthroscopy. 1994;10:558-560.

23. Pettrone FA, Gail M, Pee D, Fitzpatrick T, Van Herpe LB. Quantitative criteria for prediction of the results after displaced fracture of the ankle. J Bone Joint Surg Am. 1983;65:667-677.

24. Phillips WA, Schwartz HS, Keller CS, Woodward HR, Rudd WS, Spiegel PG, Laros GS. A prospective, randomized study of the management of severe ankle fractures. J Bone Joint Surg Am. 1985;67:67-78.

25. Ramsey PL, Hamilton W. Changes in tibiotalar area of contact caused by lateral talar shift. J Bone Joint Surg Am. 1976;58:356357.

26. Roos EM, Brandsson S, Karlsson J. Validation of the foot and ankle outcome score for ankle ligament reconstruction. Foot Ankle Int. 2001;22:788-794.

27. Takao M, Uchio Y, Naito K, Fukazawa I, Kakimaru T, Ochi M. Diagnosis and treatment of combined intra-articular disorders in acute distal fibular fractures. J Trauma. 2004;57:1303-1307.

28. Tornetta P III, Spoo JE, Reynolds FA, Lee C. Overtightening of the ankle syndesmosis: is it really possible? J Bone Joint Surg Am. 2001;83:489-492.

29. Tunturi T, Kemppainen K, Patiala H, Suokas M, Tamminen O, Rokkanen P. Importance of anatomical reduction for subjective recovery after ankle fracture. Acta Orthop Scand. 1983;54:641647.

30. van den Bekerom MP, Haverkamp D, Kloen P. Biomechanical and clinical evaluation of posterior malleolar fractures: a systematic review of the literature. J Trauma. 2009;66:279-284.

31. Weening B, Bhandari M. Predictors of functional outcome following transsyndesmotic screw fixation of ankle fractures. J Orthop Trauma. 2005;19:102-108.

32. Yde J, Kristensen KD. Ankle fractures: supination-eversion fractures of stage IV: primary and late results of operative and non-operative treatment. Acta Orthop Scand. 1980;51:981-990. 